\title{
Reconstruction becomes Informative
}

THE reconstruction of a three dimensional structure from a set of two dimensional projections is of general interest in many scientific disciplines. The problem confronting the biologist, for example, is to determine the structure of an object such as a ribosome or a virus particle from a series of electron micrographs of that object rotated about an axis. The first question that arises is how many views are necessary in order to reproduce the object to a given resolution. Second, several methods of reconstruction have been proposed and criteria are needed for choosing the best one for a particular problem. Biological objects are inherently "noisy" because intensity variations in the final image are introduced by the method of specimen preparation, non-uniformity of staining and by the effects of the electron beam itself. Another important question is, therefore, how the method of reconstruction deals with the problem of noise. This question has been difficult to answer, for proponents of reconstruction methods have tended to evaluate them using model objects.

On page 435 of this issue of Nature, Klug and Crowther have attempted to answer these questions by presenting a theoretical treatment of the reconstruction of a general object from a set of projections. They approach the problem from the standpoint of information theory, because determining the structure of an object can be regarded as equivalent to recovering a signal by sampling the information transmitted by a noisy channel.

The theory is quite general and could, at least in principle, be applied to any reconstruction method. In their article they show how the number and distribution of available projections determine the resolution for an object of a given size. In order to apply the general theory, Klug and Crowther resort to the use of Fourier transforms and reciprocal space, and although this procedure may appear somewhat prejudicial to proponents of direct space methods, they defend it on the grounds of convenience; it can be illustrated by a simple optical analogy.

Any object can be considered as a linear combination of a set of orthogonal functions. It is the coefficients of these functions that are required to obtain the three dimensional structure. The projected density distribution can, of course, be expanded in terms of these basis functions, but the coefficients that are obtained in this series cannot be used directly to reproduce the object as the basis functions themselves have been modulated by the projection and reconstruction procedure. Klug and Crowther have shown that these operations of projection and reconstruction on each basis function reproduce the function but with a different weight, and it is this weighting factor that determines the maximum possible resolution of the object even if the inherent resolution of the micrographs is greater. The number of basis functions which have significant weight and therefore contribute to the final reconstruction is determined by the number of projections available, and the value of the weighting factor is determined by the geometry of the projections. A symmetric set of equiangular projections gives rise to more significant weights than an asymmetric set.

It is at this point that the micrographs enter the picture. The coefficients of the basis functions derived from them include errors associated with the inherent noise in the object. If these errors become comparable to the weighting factor of the basis function, the inclusion of this function can produce spurious detail in the reconstructed object. This condition is more likely to occur for a highly asymmetric set of views. Conditions can thus be laid down for the determination of the number and distribution of projections necessary to obtain a given resolution for an asymmetric object of a particular size. The requirements in fact turn out to be the same as those previously obtained by Klug (Phil. Trans. Roy. Soc., B261, 173; 1971) on the basis of simpler physical arguments.

This new theory would seem to provide objective criteria for the evaluation of reconstruction methods. Whether it will put an end to the debate about the relative merits of direct space methods and Fourier methods is less clear.

\section{Bay of Fundy Tides}

In the article on page 441 of this issue of Nature, Garrett deduces a period of approximately 13.3 hours for the free mode of oscillation of the Bay of Fundy, forced by the lunar semi-diurnal tide $\left(\mathbf{M}_{2}\right)$ of the North Atlantic to produce some of the highest tidal ranges in the world. His analysis suggests that, in this mode, the whole Gulf of Maine is part of a single oscillating system containing the Bay of Fundy.

These deductions are theoretically interesting, especially in the light of some earlier work in which a different description of the development of the $\mathbf{M}_{2}$ tide was put forward. The latter work argued in favour of a near resonant mode of oscillation in the Bay extending out to the edge of the continental shelf and following essentially the route of Jordan Basin and Fundian Channel; these calculations of the effects on the tides of a barrier placed across the upper part of the Bay suggested that a decrease in tidal amplitude would be expected at the position of the barrier. On the basis of Garrett's model, however, a slight amplification of the tides seems likely in such circumstances. All this has an obvious and important relevance to the problem of estimating the tidal heights which would be available for the operation of tidal power schemes in the head waters of the Bay.

Thus Garrett's contribution, apart from introducing a new technique in interpreting tidal data, reopens the discussion on the viability of obtaining tidal power from upper Fundy, in Minas Basin and in Chignecto Bay. In many respects this discussion closed in 1969 with the publication of the report of the Atlantic Tidal Power Programming Board on the feasibility of tidal power development in the Bay of Fundy. Garrett's article provides a new element of hope that the tides in the Bay may one day be harnessed to produce electrical power. 